\title{
A Proficient and Dynamic Bidding Agent for Online Auctions
}

\author{
Preetinder Kaur, Madhu Goyal, and Jie Lu \\ DeSI lab, Centre for Quantum Computation and Intelligent Systems \\ School of Software, University of Technology, Australia \\ preetinder.kaur@student.uts.edu.au_madhu@it.uts.edu.au jielu@it.uts.edu.au
}

\begin{abstract}
E-consumers face biggest challenge of opting for the best bidding strategies for competing in an environment of multiple and simultaneous online auctions for same or similar items. It becomes very complicated for the bidders to make decisions of selecting which auction to participate in, place single or multiple bids, early or late bidding and how much to bid. In this paper, we present the design of an autonomous dynamic bidding agent (ADBA) that makes these decisions on behalf of the buyers according to their bidding behaviors. The agent develops a comprehensive method for initial price prediction and an integrated model for bid forecasting. The initial price prediction method selects an auction to participate in and then predicts its closing price (initial price). Then the bid forecasting model forecasts the bid amount by designing different bidding strategies followed by the late bidders. The experimental results demonstrated improved initial price prediction outcomes by proposing a clustering based approach. Also, the results show the proficiency of the bidding strategies amongst the late bidders with desire for bargain.
\end{abstract}

Keywords: Online auctions, Software agents, Bid forecasting, Bidding strategies, Data mining, Clustering

\section{Introduction}

The online auctions have received an extreme surge of popularity in the past few years. In the online auctions' business model, traders purchase or sell products governed by specific trading rules over the internet supporting different auction formats. There are four standard online auction formats: English auction; Dutch auction; First-price sealed-bid; and Second-price sealed-bid [1] [2]. English auctions are the most common auction type employed by the online auctioneers in eBay, Amazon etc. Bidders participating in this marketplace often face a challenge to opt for the most favorable bidding strategies to win the auction. Moreover, there are always a number of multiple auctions selling the desired item at the same time. This foster the complicated situation of the bidders in making decisions of selecting which auction to participate in, placing single or multiple bids, bidding early or late and how much to bid [3] [4]. These hard 
and time consuming processes of analyzing, selecting and making bids and monitoring are needed to be automated to assist the buyers while bidding. Software agents can promisingly act upon these tasks on behalf of the traders. These are the software tools that can execute autonomously, communicates with other agents or the user and monitors the state of its execution environment effectively. These negotiating agents outperform their human counterparts because of their systematic approach to execute complex decision making situations [5] [6]. The software agents make decisions on behalf of the consumer and endeavor to guarantee the delivery of the item according to the bidder's behavior.

In eBay auctions, a bidder with the highest value wins and he pays the second-highest maximum price. However, the bidders do not bid their maximum values either because they have trouble understanding that they should bid their maximum value or they have trouble simply figuring out what their maximum value is. Closing price prediction of the auction can help the bidders in setting their maximum valuation of the auctioned item. Furthermore, the bidders adjust their bids towards the maximum valuation of the item repeatedly in response to the remaining time left of the auction and the bids placed by the other participants, which leads to the different bidding behavior. According to different bidding behaviors, we can categorize agents as evaluators, participators, opportunists, skeptics, snipers, unmaskers or shill bidders [7] [8] [9]. Moreover, late bidding in online auctions has aroused a good deal of attention [10] [11] [12]. Late bidders appear near to the closing hours of an auction. This is a very common behavior by the bidders in an eBay style auction with hard closing rules. Late bidding may be a best response to a variety of incremental bidding strategies because they can better realize the state of the auction by observing its historical data. So there is a need to design a mechanism which decides the bid amount at particular moment of time according to the bidding behavior of the late bidders.

Predicting the closing price of an online auction is a challenging task because it depends on auction's attributes which are dynamic in nature [13]. This bid amount can be forecasted effectively by analyzing the data produced as an auction progresses (historical data). Analysis of plethora of data produced in the online auction environment can be done by using data mining techniques to predict the closing price of an online auction [14] [15]. Data from a series of the same or similar auctions closed in the past has been used to forecast the winning bid by exploiting regression, classification and regression tree, multi-class classification and multiple binary classification tasks [16] [17]. Also the history of an ongoing auction contains significant information and is exploited for the short term forecasting of the next bid by using support vector machines, functional k-nearest neighbor, clustering, regression and classification techniques [13] [15] [18]. However, these forecasted bids have hardly been used to improve the behavior of the software agents in online auctions.

This article develops an automated dynamic bidding agent (ADBA) that will use the data mining techniques to improve its behavior for bid forecasting according to different bidding strategies followed by the late bidders. ADBA 
selects an auction to participate in and predicts its closing price by adopting a clustering, and bid mapping and selection approach. Then the bidding agent models the bid amount for a given behavior based on the predicted closing price of the selected auction and the negotiation decision functions by Faratin et al [19]. These negotiation decisions are made based on the strategies generated by the bidding agent following different behaviors of the late bidders.

The rest of the paper is organized as follows. In section 2 we present the design of the Automated Dynamic Bidding Agent. Section 3 depicts experimental results evaluating the performance of the closing price prediction method and the success rate of the bidding strategies designed for different bidding behaviors of the late bidders. Section 4 concludes the paper.

\section{Automated Dynamic Bidding Agent-ADBA}

The bidding agent consists of two primary components: an initial price estimator, and a bid forecaster. The initial price estimator is responsible for the selection of an ongoing auction for participation and for predicting the closing price of the selected auction. The predicted closing price of the selected auction acts as the initial price for the bid forecaster. The bid forecaster utilizes this initial price for forecasting the bid amount for the selected ongoing auction based on different bidding strategies followed by the late bidders. The automated dynamic bidding agent (ADBA) is represented in Fig. 1.

\subsection{Initial Price Estimator}

The initial price estimator selects an auction to participate and predicts the closing price of the selected auction based on a clustering, and a bid mapping and selection approach. Formally our approach consists of four steps. First, historical data is extracted as per the requirements to form the agents' knowledge base for online auctions. Secondly, $k$-estimator agent determines the best number of partitions for the overall auction data. Thirdly, the set of similar auctions are clustered together in $k$ groups and finally, based on the transformed data after clustering and the characteristics of the current auctions, bid mapping and selection component nominates the cluster for each of the ongoing auctions to select the auction for participation, and the closing price of the selected auction will be predicted.

Data Preprocessing Let $A$ be the set of the attributes collected for each auction then $A=\left\{a_{1}, a_{2}, \ldots, a_{j}\right\}$, where $j$ is the total number of attributes. Different types of auctions are categorized based on some predefined attributes from the vast feature space of online auctions. The feature space may include average bid amount, average bid rate, number of bids, item type, seller reputation, opening bid, closing bid, quantity available, type of auction, duration of the auction, buyer reputation and many more. In this paper, to classify different types of auctions, we focus on only a set of attributes; opening 


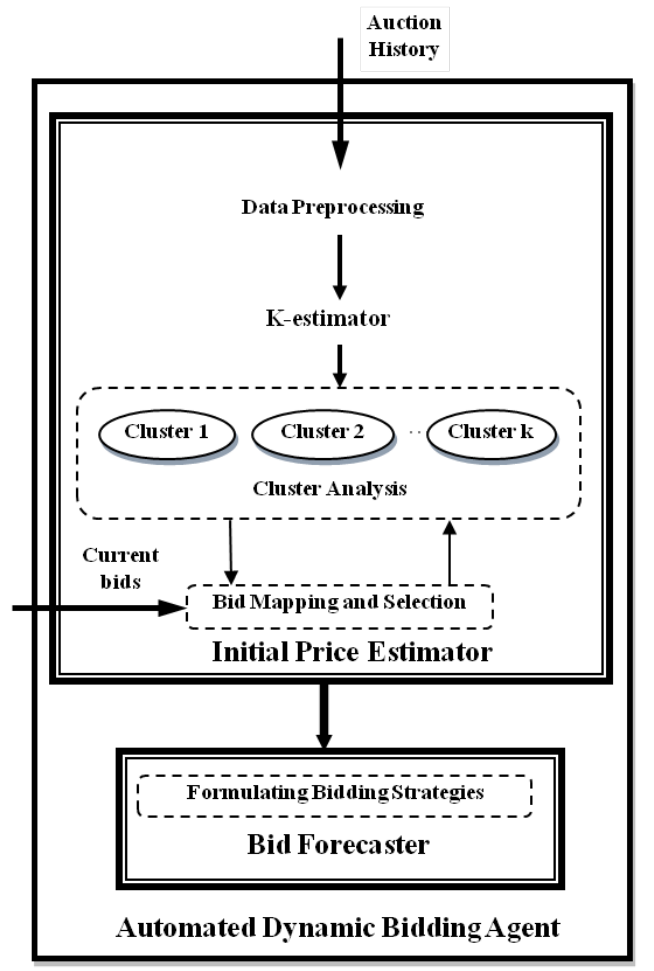

Fig. 1. Automated Dynamic Bidding Agent

bid, closing price, number of bids, average bid amount and average bid rate. Now $A=\left\{\operatorname{Open}_{i}, \operatorname{CloseP}_{i}, N U M_{i}, A v g B_{i}, A v g B R_{i}\right\}$, where $A$ be the set of attributes for an auction, $O$ pen $B_{i}$ and $C l o s e P_{i}$ be the starting price and the end price of $i^{t h}$ auction. $N U M_{i}$ be the total number of bids placed in $i^{t h}$ auction. $\operatorname{Avg} B_{i}$ be the average bid amount of $i^{\text {th }}$ auction and can be calculated as $\operatorname{Avg}\left(B_{1}, B_{2}, \ldots . . B_{l}\right)$. where $B_{1}$ be the $1^{s t}$ bid amount, $B_{2}$ be the second bid amount and $B_{l}$ be the last bid amount for $i^{\text {th }}$ auction. $A v g B R_{i}$ be the average bid rate of $i^{\text {th }}$ auction and can be calculated as

$$
\operatorname{AvgBR}_{i}=\frac{1}{n} \Sigma \frac{B_{i+1}-B_{i}}{t_{i+1}-t_{i}}
$$

where $1 \leq i \leq n, B_{i+1}$ be the amount of $(i+1)^{t h}$ bid, $B_{i}$ be the amount of $i^{t h}$ bid, $t_{i+1}$ is the time at which $(i+1)^{t h}$ bid is placed and $t_{i}$ is the time at which $i^{\text {th }}$ bid is placed.

K-estimator To decide the value of $k$ in $k$-means algorithm is a recurrent problem in clustering and is a distinct issue from the process of actually solving the clustering problem. The optimal choice of $k$ is often ambiguous, increasing 
the value of $k$ always reduce the error and increases the computation speed. The most favorable method to find $k$ adopts a strategy which balances between maximum compression of the data using a single cluster, and maximum accuracy by assigning each data point to its own cluster. In this paper the value of $k$ in $k$ - means algorithm is determined by employing elbow method using one way analysis of variance (ANOVA) [20].

Cluster Analysis A clustering based method is used to predict the closing price of the multiple ongoing auctions for autonomous agent based system. In the proposed methodology the input auctions are partitioned into groups of similar auctions depending on their different characteristics. This partitioning has been done by using $k$-means clustering algorithm [20].

Bid Mapping and Selection In order to decide that the current ongoing auctions belong to which cluster, the bid mapping and selection component is activated. Based on the transformed data after clustering and the characteristics of the current auctions, it nominates the cluster for each of the ongoing auction to select the auction to participate in and to predict its closing price.

We observe that in 92 of the 149 auctions of our dataset, winner first appear in the last hour of the auction, which account for the $62 \%$ of the total auctions, consistent with the late bidding attitude of the bidders recognized in the online auction literature [10] [11] [12]. Clustering has divided the auction data into the groups of auctions having distinct range of average bid rate $(A v g B R)$ values. It has been observed that the value of $A v g B R$ in $78 \%$ of the completed auctions belong to the same cluster as at the beginning of the last hour of the auction. So the ongoing auctions are mapped to the clusters based on their $A v g B R$ value in the beginning of the last hour. The methodology for selecting the auction for participation and predicting the closing price is described as follows:

Let $O A$ be the set of multiple ongoing auctions and $O A=O A_{1} \cup O A_{2} \cup$ $\ldots \cup O A_{k}$. where $O A_{i}$ be the set of ongoing auctions belonging to the $i^{\text {th }}$ cluster and $i=1,2, \ldots k$ where $k$ is the total number of clusters. Then $O A_{i}=$ $\left\{O A_{i l}, O A_{i 2}, \ldots O A_{i n}\right\}$ where $n$ is the total number of ongoing auctions belonging to the $i^{\text {th }}$ cluster. Let $A v g B_{i}$ be the set of average bid amounts of the ongoing auctions belonging to the $i^{t h}$ cluster at the beginning of the last hour then $A v g B_{i}=\left\{A v g B_{i l}, A v g B_{i 2}, \ldots A v g B_{i n}\right\}$. Let $A v g B C_{i}$ be the average bid amount of all the auctions in the $i^{\text {th }}$ cluster. A subset of $O A$ will be selected where $A v g B_{\text {in }}<A v g B C_{i}$, where $i=1,2, \ldots k$. Then the auction with the minimum value of the average bid amount will be selected for the participation.

The closing price prediction task for the selected auction is treated as a multiple regression task [20]. The predicted closing price of the selected auction is treated as the initial price $\left(p_{i}\right)$ by the bid forecaster.

\subsection{Bid Forecaster}

This section forecasts the bid amount for the selected ongoing auction by designing the bidding strategies for different bidding behaviors of the buyer. We 
classify the late bidders' behavior based on the single or multiple bids placements and are further categorized based on the time of the bid placement Fig. 2.

The late bidders who place single bid are Mystical and Sturdy. The mystical bidders place single bid in the last five minutes of the auction. The sturdy bidders place single bid in the last hour of the auction. The late bidders who place multiple bids are Desperate and Strategic. The desperate bidders continuously bid for an item multiple numbers of times without any other participants in between. The strategic bidders also place multiple bids to win the auction in the last hour; however, these bidders increment their bid amount strategically based on the bids placed by the other participants. The bidding strategies are designed

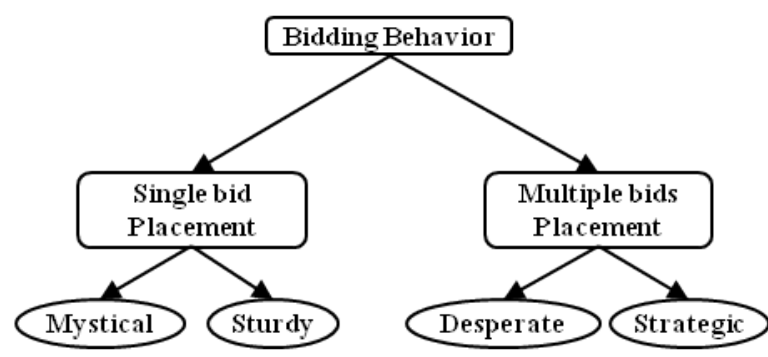

Fig. 2. Bidding Behaviors of the late bidders

for the above bidding behaviors of the buyers. The bidding agent models the bid amount based on the initial price $\left(p_{i}\right)$ and the negotiation decision functions by Faratin et al [19] for each bidding bahevior in Fig. 2. The purpose of these functions is to determine how to compute the bid amount at a particular moment of time. These negotiation decisions may depend upon the remaining time of the auction or on the bids placed by the other participants (competition).

Let $F(t)$ be the function to determine the bid amount based on the remaining time left and let $F_{c}(t)$ be the function to determine the bid amount based on the competition in the auction. Let the agent bids at time $0 \leq t \leq t_{\max }$. Agent's bidding limit is $\left[\min _{b}, \max _{b}\right]$. Then,

$$
F(t)=\min _{b}+\alpha(t)\left(\max _{b}-\min _{b}\right)
$$

where $\alpha(t)=k+(1-k)\left(\frac{\min \left(t, t_{\max }\right)}{t_{\max }}\right)^{\frac{1}{\beta}}$ A wide range of time dependent functions can be calculated by varying the value of $\alpha(t)$. where $0 \leq \alpha(0) \leq 1, \alpha(0)=k$ and $\alpha\left(t_{\max }\right)=1 k$ is a constant and $0 \leq k \leq 1$. When $k$ is multiplied by the size of the bid interval, it gives the value of the starting bid amount. $\beta$ is a constant which belongs to $R^{+}$. A number of possible bidding regulations can be obtained by varying the value of $\beta$. When $\beta<1$, minimum bid amount will be 
maintained until the $t_{\max }$ is almost reached and when $\beta>1$, agent quickly goes to its reservation price $p_{r}$ (maximum willingness to pay) where $p_{r}=p_{i}=\max _{b}$. $F_{c}(t)$ compute the bid amount at time $t$ based on the attitude of the other participants who placed the previous bids. To calculate $F_{c}(t)$ at a particular moment of time $t$, agent reproduces the behavior of the other participants $\delta \geq 1$ steps before in percentage terms. Where $n>2 \delta$.

$$
F_{c}\left(t_{n+1}\right)=\min \left(\max \left(\frac{F^{\prime}\left(t_{n-2 \delta+2}\right) F\left(t_{n-1}\right)}{F^{\prime}\left(t_{n-2 \delta}\right)}, \min _{b}\right), \max _{b}\right)
$$

Where $F^{\prime}(t)$ be the bids placed by the other participants at time t. The value of $\min _{b}$ depends on the bidding strategy followed.

Mystical Bidding Strategy In this strategy agent places single bid in the last five minutes of the auction. This bid amount depends upon the remaining time as well as the competition in the auction.

The bid amount at time $t$ for the mystical behavior will be calculated as the average of $F(t)$ as in $(2)$ and $F_{c}(t)$ in (3). Here, min $_{b}$ is the lower bound of the bid value at the start of the last five minutes of the auction. The values for $k$ and $\beta$ are set according to the behavior of the bidders. Mystical bidders commonly possess two behaviors, first, they may be desperate to get the item, and secondly, they may be willing to bargain for that item. For the mystical bidders having desperate behavior, the value of $k$ will be high and $\beta>1$, since this type of bidders bid at a price near to the reservation price $p_{r}$. On the other hand, for the mystical bidders with a desire for bargain behavior, the value of $k$ will be low and $\beta<1$.

Sturdy Bidding Strategy This strategy is similar to the mystical bidding behavior with an exception of the time of placing a bid. A bidder with sturdy behavior places a single bid at the beginning of the last hour of the auction based on the remaining time and the competition in the auction.

$F(t)$ and $F_{c}(t)$ functions similar to the mystical behavior are used to compute the bid amount, but here $\min _{b}$ is the lower bound of the bid amount at the beginning of the last hour. The sturdy bidders also appeared in two behaviors; desperate, and desire to get bargain. The values for $k$ and $\beta$ for sturdy bidders with desperate and desire to get bargain behaviors follow the same conventions as in the mystical bidders.

Desperate Bidding Strategy Desperate bidders place multiple bids continuously during the last hour of the auction. The first bid placed by the bidder depends on the remaining time left and the competition in the auction. Rest of the bids he places based on the remaining time left of the auction.

The starting bid will be calculated as the average of $F(t)$ and $F_{c}(t)$. All the other bids will be calculated from $F(t)$. The value of the $\min _{b}$ is the same as in the sturdy bidding strategy. As a desperate bidder starts bidding at a value 
close to his valuation for the item, the values for $k$ are high for this bidding strategy. Here $\beta>1$, since desperate bidders tend to quickly reach at $p_{r}$ before the deadline is reached by placing multiple bids continuously.

Strategic Bidding Strategy Strategic bidding strategy is similar to the desperate behavior with one key difference in the way of placement of the bids. Strategic bidders place each and every bid strategically based on the bids placed by the other participants in the auction. They continue bidding till the bid amount reaches their reservation price $p_{r}$.

Each bid will be calculated as the average of $F(t)$ and $F_{c}(t)$. The value of the min $_{b}$ is the same as in the sturdy bidding strategy. Strategic bidders do not start biding at an amount close to $p_{r}$; rather they increase their bid amount slowly based on the other bids in the auction. So the values for $k$ are low and $\beta<1$ for the strategic behavior.

\section{Experimentation}

The performance of our bidding agent is assessed by undertaking an empirical evaluation of the automated dynamic bidding agent (ADBA) in two phases. In the first phase, the methodology for the initial estimation is validated, and in the second phase, the success rate of the bidding agent following different behaviors of the buyers is analyzed. The dataset for our experimentation includes the complete bidding records for 149 auctions for new Palm M515 PDAs. The statistical description of the data is explained in [21].

\subsection{Initial Price Estimation}

The initial price estimator selects an auction to participate and estimates its closing price based on the clustering and bid mapping approach. This predicted closing price act as the initial price for the bid forecaster. Auction for participation is selected based on the bid mapping and selecting technique. Closing price of the selected auction is predicted by exploiting clustering and multiple linear regression approach [20]. In the proposed approach closing price of an online auction is predicted in two scenarios. First, it is predicted by exploiting multiple linear regressions on whole input auctions data. Second, by exploiting multiple linear regression on each cluster which are generated by applying $k$-means algorithm on whole input auctions data. The results are evaluated by comparing the root mean square errors (RMSEs) in both of these scenarios. Experimental results demonstrated less RMSEs for the prediction results when multiple linear regression is applied on each cluster rather than on whole input auctions data $[20]$.

\subsection{Success rate of the bidding agents}

Bid forecaster forecasts the bid amount for the selected ongoing auction based on the different bidding strategies followed by the bidder. A bidding agent with 
the bidding behavior which only depends on the $F(t)$ not on $F^{\prime}(t)$ is opted as a basis for the comparison. To compute the function $F_{c}\left(t_{n+1}\right)$, the initial values of $F^{\prime}\left(t_{n-2 \delta+2}\right), F^{\prime}\left(t_{n-2 \delta}\right)$ and $F\left(t_{n-1}\right)$ are calculated at $\delta=1$ for all the bidding strategies i.e. Mystical, Sturdy, Desperate and Strategic. For sturdy, desperate and strategic behaviors $\delta=1$ at the beginning of the last hour of the auction. For Mystical behavior, $\delta=1$ at the beginning of the last five minutes of the auction. $F^{\prime}\left(t_{n-2 \delta+2}\right)$ and $F^{\prime}\left(t_{n-2 \delta}\right)$ are calculated as the percentage of the reservation price $\left(p_{r}\right)$ reached at time $t=n-2 \delta$ and at $t=n-2 \delta+2 . F\left(t_{n-1}\right)$ is the average of $F^{\prime}\left(t_{n-2 \delta+2}\right)$ and $F^{\prime}\left(t_{n-2 \delta}\right)$. The percentage of the reservation price $\left(p_{r}\right)$ reached at time $t_{n-2 \delta+2}$ and $t_{n-2 \delta}$ for all the bidding strategies are as shown in the Table $1(\delta=1)$.

Table 1. Percentage of reservation price $\left(p_{r}\right)$ at time $t=n-2 \delta+2$ and $t=n-2 \delta$ for different bidding strategies

\begin{tabular}{|c|c|c|}
\hline Bidding Strategy & \%age of $p_{r}$ at $t_{n-2 \delta+2}$ & \%age of $p_{r}$ at $t_{n-2 \delta}$ \\
\hline Mystical & 97 & 95 \\
\hline Sturdy & 88 & 86 \\
\hline Desperate & 94 & 91 \\
\hline Strategic & 88 & 87 \\
\hline
\end{tabular}

In this set of experiments, the values for $k$ and $\beta$ depend on the selected bidding strategy for the bidding agent. Let $M S T_{d}$ and $M S T_{b}$ represent the mystical bidders with desperate and desire to bargain behavior respectively. $S T D_{d}$ and $S T D_{b}$ represent the sturdy bidders with desperate and desire to bargain behavior respectively. $D S P$ and $S T G$ represent the desperate and strategic bidders. The values for $k$ and $\beta$ chosen for these behaviors are shown in Table 2 .

Table 2. Choice of $k$ and $\beta$ for different bidding strategies

\begin{tabular}{|c|c|c|}
\hline Bidding Strategy & $k$ & $\beta$ \\
\hline$M S T_{d}$ & $0.6 \leq k \leq 1$ & $\beta>1$ \\
\hline$M S T_{b}$ & $0 \leq k \leq 0.3$ & $\beta<1$ \\
\hline$S T D_{d}$ & $0.6 \leq k \leq 1$ & $\beta>1$ \\
\hline$S T D_{b}$ & $0 \leq k \leq 0.3$ & $\beta<1$ \\
\hline$D S P$ & $0.6 \leq k \leq 1$ & $\beta>1$ \\
\hline$S T G$ & $0 \leq k \leq 0.6$ & $\beta<1$ \\
\hline
\end{tabular}

The bid amount selected by these bidding strategies depend upon the remaining time of the auction and on the bids placed by the other participants (competition). There are basically two types of bidders. They may be desperate to have the item or they may be willing to bargain to acquire the item being auctioned. Desperate bidders start bidding at higher price close to his reservation value $p_{r}$ and their bid amount is less affected by the amount of bids placed 
by the other bidders due to their desperate behavior. $M S T_{d}, S T D_{d}$, and $D S P$ bidding strategies follow this type of behavior. The bidders who are willing to bargain always bids strategically based on the bids placed by the other competitors. $M S T_{b}, S T D_{b}$ and $S T G$ follow this type of behavior. As the bidding strategies described above select the bids based on the remaining time as well as on the bids placed by the other participants (competition), these strategies will be successful when the bidder has a desire to bargain behavior. So we will discuss the performance of the agents acting strategically based on the bids placed by the other participants. The bidding agents with the behavior which only depends on the $F(t)$ not on $F^{\prime}(t)$ are opted as the basis for the comparison for each bidding strategy. The performance of all the agents following these bidding strategies is shown in Fig. 3. The performance of the agents is measured in terms of the number of times he wins the auction.

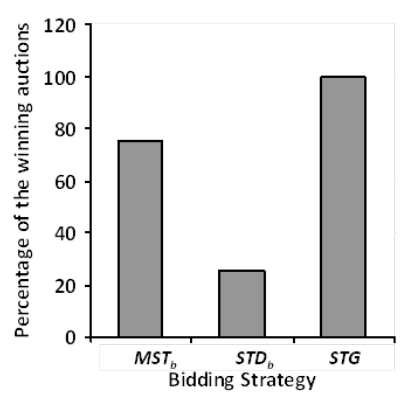

Fig. 3. Percentage of winning auction comparision

Results show that the strategy $S T G$ performed best followed by $M S T_{b}$ and then $S T D_{b}$. The agent following strategy $S T G$ has won all the auctions because he bids strategically based on the amount of the bids placed by the other participants and the remaining time of the auction. He continue bidding till he gets the item or the bid reaches at his reservation price. (In our experiments we assume that closing price of the item $\leq p_{r}$ for the agent and $p_{r}$ for the agent $\geq p_{r}$ for the competitor).

\begin{tabular}{|c|c|c|c|}
\hline \multirow{2}{*}{$\begin{array}{c}\text { Bidding } \\
\text { strategy }\end{array}$} & \multicolumn{3}{|c|}{ Desire for bargain } \\
\cline { 2 - 4 } & High & Medium & Low \\
\hline$S T G$ & $\checkmark$ & $\checkmark$ & $\checkmark$ \\
\hline$M S T_{b}$ & $\checkmark$ & $\checkmark$ & $\mathrm{X}$ \\
\hline$S T D_{b}$ & $\checkmark$ & $\mathrm{X}$ & $\mathrm{X}$ \\
\hline
\end{tabular}

Fig. 4. Choosing Bidding strategy 
To explain the performance of the agents following $M S T_{b}$ and $S T D_{b}$ strategy, the levels of the desire for bargain attitude are distinguished as low, medium and high depending on the value of $k$. It has been observed that the mystical agent wins in $75 \%$ of the auctions. The winning bidders have medium to high level of desire for bargain for having the item. The bidders with low level of desire for bargain place bids based on the remaining time of the auction. Their bid amount has been less affected by the bids of the competitors because of their attitude of single bidding in last five minutes of the auction. The agent following $S T D_{b}$ wins in $25 \%$ of the auctions. The winning bidders possess high level of desire for bargain behavior. The sturdy bidders with low and medium level of desire for bargain place bids based on the remaining time of the auction. Their bid amount has been less affected by the bids of the competitors because of their attitude of single bidding at the beginning of the last hour of the auction. The most suitable bidding strategy based on the desire of the bargain for the bidder has been shown in Fig. 4.

\section{Conclusions}

In this paper we presented an automated dynamic bidding agent (ADBA) that uses data mining techniques to improve its behavior for bid forecasting according to different bidding strategies followed by late bidders. The bidding agent primarily performs three tasks; first, it decides which auction to bid in, secondly, predicts the closing price of the selected auction, and finally, models the bid amount for a given bidding behavior of the bidders. Auction selection and the closing price prediction adopt a clustering, and bid mapping and selection approach. The ADBA forecasts the bid amount for the selected ongoing auction by designing the bidding strategies based on the bidding behavior of the bidders. Bidding strategies are designed according to the predicted closing price and the negotiation decision functions for the specific bidding behaviors. By allowing negotiation decision functions for different bidding behaviors of the buyers, ADBA presents improved bid forecasting results than the DC mechanism [15]. The outcome of the clustering based model for the closing price prediction is compared with the classic model for price prediction. The improvement in the error measure for each cluster for a set of attributes gives support in favor of the proposed model using clustering. Our experimental results demonstrated the proficiency of the designed bidding strategies for the late bidders with different levels of desire for bargain behavior.

\section{References}

1. Ockenfels, A., D.H. Reiley Jr, A. Sadrieh: Online auctions. National Bureau of Economic Research Cambridge, Mass., USA (2006)

2. Haruvy, E.: Internet auctions. Foundations and Trends in Marketing, vol. 4, issue. 1, pp. 1-75 (2009) 
3. Park, Y.H., E.T. Bradlow: An integrated model for bidding behavior in Internet auctions: Whether, who, when, and how much. In: Journal of Marketing Research, vol. 42 , issue 4 , pp. 470-482 (2005).

4. Jank, W., S. Zhang: An automated and data-driven bidding strategy for online auctions. In: Journal of Computing, vol. 23, issue 2, pp. 238-253 (2011).

5. Anthony, P., et al.: Autonomous agents for participating in multiple online auctions. In: IJCAI Workshop on E-Business and the Intelligent Web, Seattle, USA, pp. 54-64 (2001)

6. Greenwald, A., P. Stone: Autonomous bidding agents in the trading agent competition. In: IEEE Internet Computing, vol. 5, issue 2, pp. 52-60 (2001)

7. Bapna, R., et al.: User heterogeneity and its impact on electronic auction market design. In: An empirical exploration. Mis Quarterly, vol. 28, issue 1, pp. 21-43 (2004)

8. Shah, H., et al.: Mining eBay: Bidding strategies and shill detection. In: WEBKDD 2002-MiningWeb Data for Discovering Usage Patterns and Profiles, pp. 17-34 (2003)

9. Trevathan, J., W. Read: Detecting shill bidding in online English auctions. In: Handbook of Research on Social and Organizational Liabilities in Information Security (2008)

10. Du, L., Q. Chen, N. Bian: An Empirical Analysis of Bidding Behavior in Simultaneous Ascending-Bid Auctions. In: International Conference on E-Business and E-Government (ICEE), IEEE (2010)

11. Rasmusen, E.B., Strategic implications of uncertainty over one's own private value in auctions. In: The BE Journal of Theoretical Economics, vol. 6,pp-. 7(2006)

12. Ockenfels, A., A.E. Roth: Late and multiple bidding in second price Internet auctions: Theory and evidence concerning different rules for ending an auction. In: Games and Economic Behavior, vol. 55, issue 2 pp. 297-320 (2006).

13. Xuefeng, L., et al.: Predicting the final prices of online auction items. In: Expert Systems with Applications, vol. 31, issue 3, pp. 542-550 (2006).

14. Nikolaidou, V., P. Mitkas: A Sequence Mining Method to Predict the Bidding Strategy of Trading Agents. In: Agents and Data Mining Interaction. p p. 139-151 (2009)

15. Kehagias, D.D.,P.A. Mitkas: Efficient E-Commerce Agent Design Based on Clustering eBay Data. In: International Conferences on Web Intelligence and Intelligent Agent Technology Workshops, IEEE/WIC/ACM ( 2007)

16. Heijst, D., R. Potharst, M. Wezel: A support system for predicting ebay end prices. Econometric Institute Report (2006)

17. Ghani, R., H. Simmons: Predicting the end-price of online auctions ( 2004).

18. Zhang, S., W. Jank, G. Shmuel: Real-time forecasting of online auctions via functional k-nearest neighbors. In: International Journal of Forecasting, vol. 26, issue 4pp. 666-683 (2010)

19. Faratin, P., C. Sierra, N.R. Jennings: Negotiation decision functions for autonomous agents. In: Robotics and Autonomous Systems, vol. 24 pp. 159-182 (1998)

20. Kaur, P., M. Goyal, J. Lu: Data mining driven agents for predicting online auction's end price. In IEEE Symposium on Computational Intelligence and Data Mining (CIDM). IEEE, Paris, pp. 141-147 (2011)

21. Kaur, P., M. Goyal, J. Lu: Pricing Analysis in Online Auctions Using Clustering and Regression Tree Approach. In: Agents and Data Mining Interaction, L. Cao, et al., Editors., Springer Berlin / Heidelberg. pp. 248-257 (2012) 